\title{
Corrigendum
}

Martina Zaninotto and Mario Plebani*

\section{Corrigendum to: Understanding and managing interferences in clinical laboratory assays: the role of laboratory professionals}

https://doi.org/10.1515/cclm-2021-0212

Corrigendum to: Zaninotto $\mathrm{M}$, Plebani $\mathrm{M}$. Understanding and managing interferences in clinical laboratory assays: the role of laboratory professionals. Clin Chem Lab Med 2020;(3):1005-1005. https://doi.org/10.1515/cclm-2019-0898.

Unfortunately, the article contains two typos both in the Abstract and in the section "Frequency and type of interferences": in two sentences it reads "... that are not undetectable before analysis ...", however, "not" is mistakenly included in the sentences.
The correct sentences should read:

The first (type 1) is represented by lipemia, hemolysis and icterus, and the second (type 2), by unusual constituents that are undetectable before analysis, and may affect the matrix of serum/plasma of individual subjects.

The first (type 1), easy to detect, is represented by lipemia, hemolysis and icterus, and the second (type 2), by unusual constituents that are undetectable before analysis, and may affect the matrix of serum/plasma of individual subjects.

*Corresponding author: Prof. Mario Plebani, Department of Laboratory Medicine, University-Hospital of Padova, Padova, Italy, Phone: +39 0498212792, E-mail: mario.plebani@unipd.it. https:// orcid.org/0000-0002-0270-1711

Martina Zaninotto, Department of Laboratory Medicine, UniversityHospital of Padova, Padova, Italy 